\title{
A Computational Analysis of Two-Phase Casson Nanofluid Passing a Stretching Sheet Using Chemical Reactions and Gyrotactic Microorganisms
}

\author{
Zulqurnain Sabir, ${ }^{1,2}$ Rizwan Akhtar ${ }^{\mathbb{D}},{ }^{1}$ Zhu Zhiyu, ${ }^{1}$ Muhammad Umar, ${ }^{2}$ Ali Imran, ${ }^{3}$ \\ Hafiz Abdul Wahab $\mathbb{D}^{2},{ }^{2}$ Muhammad Shoaib, ${ }^{3}$ and Muhammad Asif Zahoor Raja ${ }^{4}{ }^{4}$ \\ ${ }^{1}$ School of Electronics and Information, Jiangsu University of Science and Technology, Zhenjiang, China \\ ${ }^{2}$ Department of Mathematics and Statistics, Hazara University, Mansehra (KPK), Pakistan \\ ${ }^{3}$ Department of Mathematics, COMSATS University Islamabad, Attock Campus, 43600 Attock, Pakistan \\ ${ }^{4}$ Department of Electrical and Computer Engineering, COMSATS University Islamabad, Attock Campus, 43600 Attock, Pakistan \\ Correspondence should be addressed to Rizwan Akhtar; rizwan@just.edu.cn
}

Received 19 March 2019; Revised 9 May 2019; Accepted 26 May 2019; Published 16 June 2019

Academic Editor: Mohamed Abd El Aziz

Copyright (C) 2019 Zulqurnain Sabir et al. This is an open access article distributed under the Creative Commons Attribution License, which permits unrestricted use, distribution, and reproduction in any medium, provided the original work is properly cited.

In this study, an attempt is made to explore the two-phase Casson nanofluid passing through a stretching sheet along a permeable surface with the effects of chemical reactions and gyrotactic microorganisms. By utilizing the strength of similarity transforms the governing PDEs are transformed into set of ODEs. The resulting equations are handled by using a proficient numerical scheme known as the shooting technique. Authenticity of numerical outcomes is established by comparing the achieved results with the MATLAB built-in solver bvp4c. The numerical outcomes for the reduced Nusselt number and Sherwood number are exhibited in the tabular form, while the variations of some crucial physical parameters on the velocity, temperature, and concentration profiles are demonstrated graphically. It is observed that Local Nusselt number rises with the enhancement in the magnetic field parameter, the porous media parameter, and the chemical reactions, while magnetic field parameter along with porous media parameter retards the velocity profile.

\section{Introduction}

The study of two-phase nanofluids has taken considerable hype due to its vast applications in the domain of science and technology, industrial studies, engineering, and biomedical engineering [1]. Due to utmost importance of the Casson nanofluid problems, several methodologies have been employed to enhance the thermal appearances of the regular fluids. Das [2] used the convective slip, for analyzing heat properties of nanofluids fluid through a porous stretched surface. Khan et al. [3] discovered the dynamics of a nanofluid through boundary-layer region over a stretching surface. Gorla and Chamkha [4] solved the nanofluid flow problem over a horizontal plate using a permeable surface along natural convective boundary-layer conditions through a horizontal plate. Noghrehabadi et al. [5] explored the physics of heat transfer by employing slip in the boundary layer region for the flow of a nanofluid together with dynamic nanoparticles. In another study Noghrehabadi et al. [6] observed decline in the Nusselt number when slip factor is enhanced. Ibrahim and Shanker [7] investigated the flow of nanofluid in boundary layer region along with heat transfer effect past a porous stretching surface by incorporating magnetic field properties, slip at boundary, and thermal radiation. Goyal and Bhargava [8] reported the heat transfer flow of viscoelastic nanofluid with velocity slip at boundary past a stretched surface. Recently, nanofluid flow along stretching sheet with Lorentz's impact has taken huge inspiration for the researchers' community because of its significant involvement in MHD generators, nuclear reactor studies, plasma physics, wire drawing, manufacturing of glass fiber and hot rolling, etc. 
Recently the flow involving heat and mass conversion investigations on fluids along with chemical effects past through stretching surface has proven significant importance in metallurgy and industrial studies of chemical engineering like polymer production and food processing. Some possible applications are the study of drying, temperature and moisture distribution, decline of temperature in a cooling tower, and desert cooler flow [9]. The traditional fluids, namely, water, oil, and ethylene glycol compound, are considered very weak fluids for the transfer of heat. The heat involvement through the surface and the medium is strongly affected by the thermal conductivity. Sheikholeslami et al. [10] used the radiation effects for free convective heat transfer and they recovered that the intensity of heat transfer reduces due to the Lorentz forces. Garoosi et al. [11] reported the combined convection and naturally convective heat transfer between heated cylinders of differential length filled with nanofluid. Turkyilmazoglu [12] investigated mathematically MHD fluid flow through porous surface with heat involvement persuaded by stretching or shrinking sheet for the two and three dimensional deformed objects. Boungiorno [13] worked on convective dynamics of nanofluids by taking Brownian diffusion and established the unnatural convective heat transfer viewed in nanofluids. He also explained the importance of two nanoparticles, Brownian diffusion and thermophoresis. Congedo et al. [14] with the aid of finite volume method calculated the numerical results for naturally convective heat transfer nanofluids flow. Rashidi et al. [15] made investigations to explore the flow of nanofluid within permeable rotating disk in the presence of magnetic field by employing the law of thermodynamics. They also explained the importance of the magnetic disk, in the application of heat expansion development for long lasting energy systems.

The problems of porous medium have attained much consideration of the researchers due to its vast utilities in heat exchange, water-flow in reservoirs, rock mechanics, acoustics study, soil mechanics, production of oil, and catalytic reactors. The porous media play a crucial role in increasing the overall efficiency of the solar systems, improving the heating process of green houses and in storing the solar heat in packed beds. Vafai [16] investigated the forced convection flow in a permeable channel and achieved the expressions for velocity and temperature profiles with the help of exact solution. They used the porous medium properties and considered the fluid to be homogeneous and isotropic. Nakayama et al. [17] used the non-Darcy forced convective heat transfer in a medium restricted by channel boundaries. Nield et al. [18] presented an analytical technique to formulate the forced convection in a plane working on a saturated bidispersed permeable channel. They observed that with the finite thermal resistance effects reduction happens in the intensity of unstable local thermal and transfer of heat to the porous medium. Bakier [19] used the Runge-Kutta method while studying the impacts of thermal radiation on the mixed convection in a permeable geometry.

The bioconvection flow is the large scale convection of fluid flow produced by the density gradient formed by swimming of dynamic microorganisms. Oberbeck-Boussinesq calculation with aid of the similarity transformation for MHD boundary layer flow with transfer of heat and mass in a water-oriented nanofluid having gyrotactic microorganisms passing through a transverse plate was reported by Khan et al. [20]. Xu et al. [21] used the mixed convection flow passing on a stretching sheet with both gyrotactic microorganisms and nanoparticles. They found that the thermophoresis parameter rises as the Nusselt number declines. Kuznetsov [22] presented the commencement of bioconvection having a nanofluid and gyrotactic microorganisms. To find an analytical solution, Galerkin and a linear instability method were used for the nonoscillatory condition. Kuznetsov and Nield [23] proposed the model of mixed bioconvection and later on this model was studied by $\mathrm{Xu}$ and Pop [24]. Mahdy [25] discussed a transverse cone in a permeable media, when bioconvection in a peristaltic flow takes place in an asymmetric channel that is comprised of nanofluid enclosing the gyrotactic microorganisms. Akbar [26] reported the bionano-engineering model by using bioconvection peristaltic flow in a channel of asymmetric geometry using nanofluid comprising gyrotactic microorganisms.

The electrically conducting two-dimensional steady incompressible viscous nanofluid flow passing a stretching sheet has diverse applications in the industry. Some of the key applications of viscous flow through a stretching sheet [27-31] can be narrated as aerodynamic extrusion of plastic sheets, condensation mechanism of metallic plate through a cooling bath, and manufacturing of a polymer sheet through a dye, processing of food stuffs, glass fiber, metallic packaging, rope rolling, and aluminum jug industrial advancements, etc.

The prime features of current study are described as follows:

(i) A novel mathematical model is developed for twophase Casson nanofluid through a stretching sheet on a permeable surface with the variations of chemical reactions and gyrotactic microorganisms.

(ii) By utilizing the strength of similarity transforms the resulting PDEs are changed into set of ODEs.

(iii) The resulting ODEs are tackled by using a well established proficient numerical scheme known as the shooting technique.

(iv) Numerical results are validated by comparing the achieved results with the MATLAB built-in solver bvp4c.

(v) Numerical outcomes for the reduced Nusselt number and Sherwood number are exhibited in the tabular form while the impacts of crucial parameters are investigated graphically.

\section{Mathematical Interpretation of the Problem}

A novel electrically conducting two-dimensional steady incompressible viscous nanofluid flow is investigated through a stretching surface having plate temperature $T_{w}>T_{\infty}$ and species concentration $C_{w}>C_{\infty}$, respectively. The stretching sheet is taken along $x$-axis in the direction of the motion and $y$-axis is taken in the transverse direction of the plate. The 


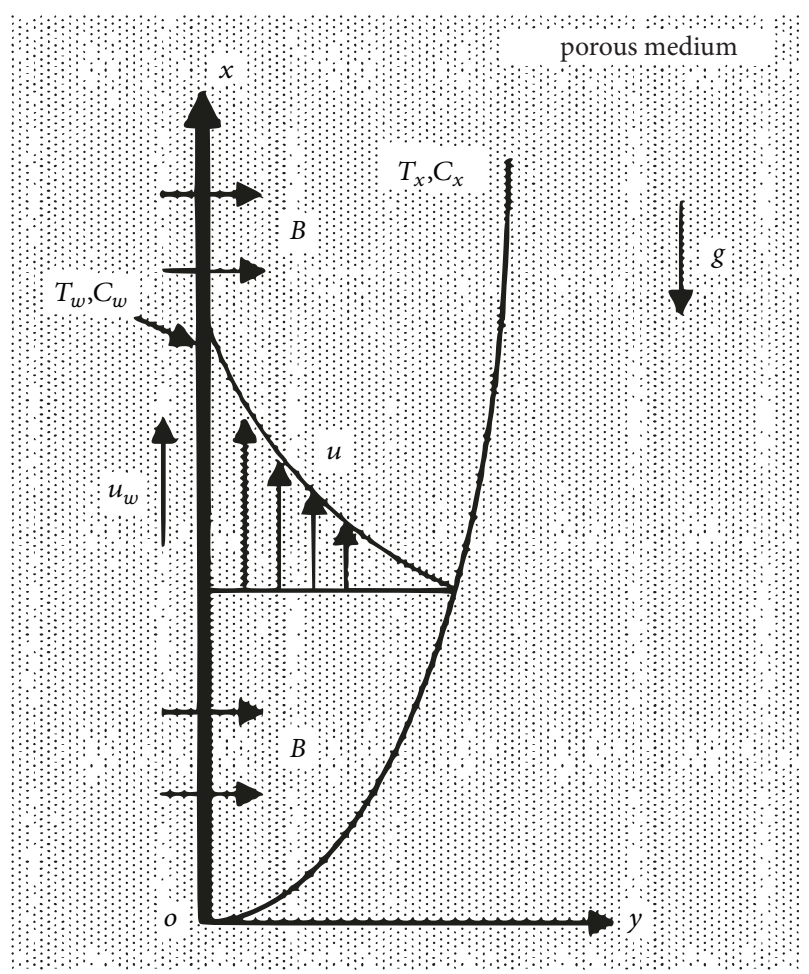

FIGURE 1: Geometrical interpretation of the problem.

elementary equations for momentum, energy, concentration, and gyrotactic microorganism [32,33] are described as in [32] while the coordinate setting and physical configuration are exhibited in Figure 1.

$$
\begin{aligned}
& \frac{\partial u}{\partial x}+ \frac{\partial v}{\partial y}=0 \\
& u \frac{\partial u}{\partial x}+ v \frac{\partial u}{\partial y} \\
&= \tilde{v}\left(1+\frac{1}{\delta}\right) \frac{\partial^{2} u}{\partial y^{2}}-\frac{v}{K} u-c^{*} \varepsilon^{2} u^{2}-\frac{\sigma B_{0}^{2}}{\rho_{f}} u \\
&+g \beta\left(T-T_{\infty}\right)+g \beta^{*}\left(C-C_{\infty}\right) \\
& u \frac{\partial T}{\partial x}+ \frac{\partial T}{\partial y} \\
&= \alpha \frac{\partial^{2} T}{\partial y^{2}}-\frac{\widetilde{v}}{C p}\left(\frac{\partial u}{\partial y}\right)^{2} \\
&+\tau\left[D_{B} \frac{\partial C}{\partial y} \frac{\partial T}{\partial y}+\left(\frac{D_{T}}{T_{\infty}}\right)\left(\frac{\partial T}{\partial y}\right)^{2}\right] \\
&+\frac{Q^{*}}{\rho C p}\left(T-T_{\infty}\right) \\
& u \frac{\partial C}{\partial x}+v \frac{\partial C}{\partial y}=D_{B} \frac{\partial^{2} C}{\partial y^{2}}+\left(\frac{D_{T}}{T_{\infty}}\right) \frac{\partial^{2} T}{\partial y^{2}}-K_{1}\left(C-C_{\infty}\right)
\end{aligned}
$$

$$
\begin{aligned}
& u \frac{\partial N}{\partial x}+v \frac{\partial N}{\partial y}+\frac{b W c}{\left(C_{w}-C_{\infty}\right)}\left[\frac{\partial}{\partial y}\left(N \frac{\partial C}{\partial y}\right)\right] \\
& =D_{m} \frac{\partial^{2} N}{\partial y^{2}} .
\end{aligned}
$$

The suggested boundary conditions are written as

$$
\begin{aligned}
& u=u_{w}(x)=u_{0} e^{x / l}, \\
& v=0 \text {, } \\
& T=T_{w}=T_{\infty}+T_{0} e^{x / 2 l}, \\
& C=C_{w}=C_{\infty}+C_{0} e^{x / 2 l}, \\
& N=N_{w}=N_{\infty}+N_{0} e^{x / 2 l} \\
& \text { at } y=0 \text {, }
\end{aligned}
$$

as $y \longrightarrow \infty$.

The nonuniform permeability of the source is taken as $K=$ $k e^{x / l}$ whereas the dimensional heat and chemical reaction parameters were taken as $Q^{*}=Q_{0} e^{x / l}$ and $K=K_{0} e^{x / l}$, respectively. The similarity transformations are given as

$$
\begin{aligned}
u & =u_{0} e^{x / l} f^{\prime}(\eta), \\
v & =-\sqrt{\frac{\nu u_{0}}{2 l}} e^{x / 2 l}\left[f(\eta)+\eta f^{\prime}(\eta)\right] \\
\eta & =y \sqrt{\frac{u_{0}}{2 \nu l}} e^{x / 2 l} \\
\theta(\eta) & =\frac{T-T_{\infty}}{T_{w}-T_{\infty}} \\
S(\eta) & =\frac{C-C_{\infty}}{C_{w}-C_{\infty}} \\
\varepsilon(\eta) & =\frac{N-N_{\infty}}{N_{w}-N_{\infty}} \\
I & =l c^{*} \zeta^{2} .
\end{aligned}
$$

Using the above transformations, the ordinary differential equations (ODEs) are given below:

$$
\begin{aligned}
& V_{r}\left(1+\frac{1}{\beta}\right) f^{\prime \prime \prime}-2(M+k) f^{\prime}-2(I+1)\left(f^{\prime}\right)^{2} \\
& \quad+f f^{\prime \prime}+2 \lambda_{T} \theta+2 \lambda_{M} S=0,
\end{aligned}
$$




$$
\begin{aligned}
& \theta^{\prime \prime}-\operatorname{Pr} f^{\prime} \theta+\operatorname{Prf} \theta^{\prime}+\operatorname{PrEc} V_{r}\left(f^{\prime \prime}\right)^{2} \\
& +\operatorname{Pr}\left[N b S^{\prime} \theta^{\prime}+N t\left(\theta^{\prime}\right)^{2}\right]+2 \operatorname{PrQ} \theta=0, \\
& S^{\prime \prime}-L e\left(f^{\prime} S+2 \chi S-f S^{\prime}\right)+\frac{N t}{N b} \theta^{\prime \prime}=0, \\
& \epsilon^{\prime \prime}+L b f \epsilon^{\prime}-P e\left(\epsilon^{\prime} S^{\prime}+(\epsilon+\Omega) S^{\prime \prime}\right)=0 .
\end{aligned}
$$

The boundary conditions may be written in the dimensionless form as

$$
\begin{aligned}
& f^{\prime}(\eta)=1, \\
& f(\eta)=0, \\
& \theta(\eta)=1, \\
& S(\eta)=1, \\
& \epsilon(\eta)=1 \\
& f^{\prime}(\eta)=0, \\
& \theta(\eta)=0, \\
& S(\eta)=0, \\
& \epsilon(\eta)=0
\end{aligned}
$$

In the above equations, different parameters have the following formulations:

$$
\begin{aligned}
V_{r} & =\frac{\tilde{v}}{v} \\
\lambda_{T} & =\frac{\operatorname{Lg} \beta\left(T_{w}-T_{\infty}\right)}{u_{0}^{2} e^{2 x / l}}, \\
\lambda_{M} & =\frac{L g \beta^{*}\left(C_{w}-C_{\infty}\right)}{u_{0}^{2} e^{2 x / l}}, \\
P e & =\frac{b W c}{D_{m}}, \\
\Omega & =\frac{N_{\infty}}{N_{w}-N_{\infty}}, \\
M & =\frac{l \sigma B_{0}^{2}}{u_{0} \rho_{f} e^{x / l}}, \\
P r & =\frac{v}{\alpha}, \\
N b & =\frac{\tau D_{B}}{v}\left(C_{w}-C_{\infty}\right), \\
N t & =\frac{\tau D_{t}}{v T_{\infty}}\left(T_{w}-T_{\infty}\right),
\end{aligned}
$$

$$
\begin{aligned}
k & =\frac{L v}{u_{0} K e^{x / l}}, \\
L e & =\frac{v}{D_{B}}, \\
Q^{*} & =\frac{L Q_{0}}{u_{0} \rho c_{p}}, \\
\chi & =\frac{l K_{0}}{u_{0}}, \\
E c & =\frac{u_{w}^{2}}{c_{p}\left(T_{w}-T_{\infty}\right)}, \\
L b & =\frac{\nu}{D_{m}}, \\
\lambda & =\frac{L \beta g \rho f_{\infty}\left(1-C_{\infty}\right)\left(T_{w}-T_{\infty}\right)}{\rho_{f} u_{0}^{2} e^{2 x / l}}, \\
R b & =\frac{g \gamma\left(N_{w}-N_{\infty}\right)\left(\rho_{m}-\rho_{f}\right)}{\rho_{f} u_{0}^{2} e^{2 x / l}}, \\
\rho_{f} u_{0}^{2} e^{2 x / l} & \frac{L g\left(C_{w}-C_{\infty}\right)\left(\rho_{f}-\rho_{f_{\infty}}\right)}{} .
\end{aligned}
$$

\section{Solution Methodology}

A brief description on the numerical approach used for the solution of the above nonlinear, nondimensional, and coupled ODEs (8)-(11) along with the boundary conditions (12) has been presented in this section. The nonlinear systems of equations are tackled with aid of well known numerical technique, namely, the shooting method. For comparison purpose, the same system is solved by using the MATLAB built-in solver bvp4c. The domain has been taken as $\left[0, \eta_{\max }\right]$. Due to the observed ignorable variations in the solution for $\eta>10, \eta_{\max }$ has been chosen as 10 throughout the article. To convert the system of higher order ODEs given in Eqs. (8)(11) to set of first order ODEs, $f$ is denoted by $y_{1}, \theta$ by $y_{4}, S$ by $y_{6}$, and $\epsilon$ by $y_{8}$. The resulting equations are written as

$$
\begin{aligned}
y_{1}^{\prime} & =y_{2}, \\
y_{2}^{\prime} & =y_{3}, \\
y_{3}^{\prime} & =\frac{\beta}{(\beta+1) V_{r}}\left(2(M+k) y_{2}+2(I+1) y_{2}^{2}-y_{1} y_{3}\right. \\
& \left.-2 \lambda y_{4}+2 N r y_{6}+2 R b y_{8}\right), \\
y_{4}^{\prime} & =y_{5}, \\
y_{5}^{\prime} & =\operatorname{Pr}\left(y_{2} y_{4}-y_{1} y_{5}-E c V_{r} y_{3}^{2}\right. \\
& \left.-\left[N b y_{5} y_{7}+N t y_{5}^{2}\right]-2 Q y_{4}\right),
\end{aligned}
$$


TABLE 1: The variations of $\operatorname{Pr}$ and $E c$ are demonstrated with $\lambda=N r=M=R b=k=L e=I=Q=\chi=N t=\Omega=0, N t=0.001$.

\begin{tabular}{llccc}
\hline & & Ref. [27] & Ref. [28] & Present \\
\hline $\operatorname{Pr}$ & $E c$ & $-\theta^{\prime}(0)$ & $-\theta^{\prime}(0)$ & $-\theta^{\prime}(0)$ \\
\hline 1 & 0.0 & 0.9550 & 0.9547 & 0.9562 \\
2 & 0.0 & 1.4714 & 1.4719 & 1.4728 \\
3 & 0.0 & 1.8691 & 1.8701 & 1.8704 \\
1 & 0.2 & 0.8622 & 0.8629 & 0.8643 \\
2 & 0.2 & 1.3055 & 1.3062 & 1.3080 \\
3 & 0.2 & 1.6882 & 1.6890 & 1.6411 \\
1 & 0.9 & 0.5385 & 0.5392 & 0.5426 \\
2 & 0.9 & 0.7248 & 0.7250 & 0.7311 \\
3 & 0.9 & 0.8301 & 0.8309 & 0.8386 \\
\hline
\end{tabular}

$$
\begin{aligned}
& y_{6}^{\prime}=y_{7}, \\
& y_{7}^{\prime}=\operatorname{Le}\left(y_{2} y_{6}+2 \chi y_{6}-y_{1} y_{7}\right)-\frac{N t}{N b} y_{5}^{\prime}, \\
& y_{8}^{\prime}=y_{9}, \\
& y_{9}^{\prime}=-\operatorname{Lb} y_{1} y_{9}+\operatorname{Pe}\left(y_{7}^{\prime} y_{8}+\Omega y_{7}^{\prime}+y_{7} y_{9}\right) .
\end{aligned}
$$

With the new notations, the set of initial conditions from (12) gets the following form:

$$
\begin{aligned}
& y_{1}(0)=0, \\
& y_{2}(0)=1, \\
& y_{4}(0)=1, \\
& y_{6}(0)=1, \\
& y_{8}(0)=1 .
\end{aligned}
$$

By choosing the missing initial conditions $y_{3}(0)=s, y_{5}(0)=$ $t, y_{7}(0)=w$ and $y_{9}(0)=p$, the resulting initial value problem is obtained by the shooting method. Newton's method has been opted to update the missing initial conditions. The numerical solution is gathered by defining following accuracy criteria:

$$
\begin{aligned}
& \max \left\{\left|y_{2}\left(\eta_{\max }\right)\right|,\left|y_{4}\left(\eta_{\max }\right)\right|,\left|y_{6}\left(\eta_{\max }\right)\right|,\left|y_{8}\left(\eta_{\max }\right)\right|\right\} \\
& \quad<\xi
\end{aligned}
$$

where $\xi>0$ is very small and positive number. The numerical outcomes of this work are obtained with $\xi=10^{-6}$.

A critical analysis of present investigation with already obtained result available in the literature for $\theta^{\prime}(0)$ against the Prandtl number $\mathrm{Pr}$ and Eckert number $E c$ with those of Ferdows et al. [34] and Bidin and Nazar [35] is shown in Table 1. The computed result exhibited in Table 1 shows impressive match with the already available results.

\section{Results and Discussions}

It is quite clear from Table 2 that the amount of heat transfer reduces with the rise in magnetic parameter $M$, nonuniform permeability $k$, dimensionless inertia factor $I$, mass convective parameter $\lambda_{M}$, Eckert number $E c$, thermophoresis factor $N t$, and Brownian motion factor $N b$; on other hand quite different behavior is noticed for the thermal convective parameter $\lambda_{T}$ and Prandtl parameter $\operatorname{Pr}$. However Sherwood number is found to have a decreasing impact due to the magnetic parameter $M$, nonuniform permeability $k$, dimensionless inertia factor $I$, and Prandtl parameter $\mathrm{Pr}$, while an opposite behavior is seen for the thermal convective parameter $\lambda_{T}, \lambda_{M}$ which is the mass convective parameter, Eckert number $E c$, thermophoresis factor $N t$, and Brownian motion factor $\mathrm{Nb}$.

Table 3 demonstrates the behavior of local Nusselt number values and Sherwood number values for varying various flow indicators with $\lambda_{M}=0, M=k=I=\lambda_{T}=E c=N t=$ $N b=0.1$, and $\operatorname{Pr}=1$. The rate of heat transfer increases for the heat generation/absorption $Q$ and viscosity ratio factor $V_{r}$ while an opposite behavior is noticed for Lewis number Le, chemical reaction factor $\chi$, and Casson fluid factor $\beta$. Furthermore, Sherwood number increases for the Lewis number $L e$, chemical reaction factor $\chi$, and viscosity ratio factor $V_{r}$ and decreases for the heat generation/absorption parameter $Q$ and Casson fluid factor $\beta$.

In order to have a deep insight of the solution, graphical illustrations of different profiles are presented. Figure 2 reflects the behavior of velocity profile for various values of the magnetic field parameter $M$. The magnetic field along with the electric current generates a force which is reputed as Lorentz force creates obstacle in the fluid motion, which results in decrements in the velocity profile. Since the magnetic force is applied against the direction of the flow which raises stationary fluid absorption on the stretching sheet also retards the flow momentum. As a result, the flow becomes slow. Figure 3 speculates the conduct of the velocity profile against the porous media parameter $k$. The porosity parameter escalates when the fluid becomes more viscous. Due to an enhancement in the viscosity, the velocity of the fluid is declined. The graphical impact of the dimensionless inertia parameter $I$ is demonstrated in Figure 4. It is quite evident that, by enhancing the inertia parameter, the thermal boundary layer thickness reduces, which creates a decrement in the velocity profile. Analysis of increasing Prandtl number $\operatorname{Pr}$ value on velocity profile is exhibited in Figure 5. The 
TABLE 2: The variations of Local Nusselt number and Sherwood number for different flow parameters.

\begin{tabular}{|c|c|c|c|c|c|c|c|c|c|c|c|c|}
\hline & & & & & & & & & \multicolumn{2}{|c|}{$N u_{x} R e_{x}^{-1 / 2}$} & \multicolumn{2}{|c|}{$S h_{x} R e_{x}^{-1 / 2}$} \\
\hline$M$ & $k$ & $I$ & $\lambda_{T}$ & $\lambda_{M}$ & $\mathrm{Pr}$ & $E c$ & $N t$ & $\mathrm{Nb}$ & shooting & bvp4c & shooting & bvp4c \\
\hline 0.1 & 0.1 & 0.1 & 0.1 & 0 & 1 & 0.1 & 0.1 & 0.1 & 0.9690 & 0.9690 & 0.1681 & 0.1681 \\
\hline 0.2 & & & & & & & & & 0.9569 & 0.9569 & 0.1646 & 0.1646 \\
\hline 0.3 & & & & & & & & & 0.9453 & 0.9453 & 0.1617 & 0.1617 \\
\hline 0.4 & & & & & & & & & 0.9341 & 0.9341 & 0.1593 & 0.1593 \\
\hline & 0.5 & & & & & & & & 0.9232 & 0.9232 & 0.1573 & 0.1573 \\
\hline & 1 & & & & & & & & 0.8736 & 0.8736 & 0.1523 & 0.1523 \\
\hline & 1.5 & & & & & & & & 0.8297 & 0.8297 & 0.1501 & 0.1501 \\
\hline & & 0.5 & & & & & & & 0.9473 & 0.9473 & 0.1664 & 0.1664 \\
\hline & & 1 & & & & & & & 0.9230 & 0.9230 & 0.1657 & 0.1657 \\
\hline & & 1.5 & & & & & & & 0.9013 & 0.9013 & 0.1650 & 0.1650 \\
\hline & & & 0.5 & & & & & & 0.9999 & 0.9999 & 0.1731 & 0.1731 \\
\hline & & & 1 & & & & & & 1.0313 & 1.0313 & 0.1786 & 0.1786 \\
\hline & & & 1.5 & & & & & & 1.0574 & 1.0574 & 0.1837 & 0.1837 \\
\hline & & & & 0.5 & & & & & 0.9133 & 0.9133 & 0.1324 & 0.1324 \\
\hline & & & & 1 & & & & & 0.7437 & 0.7437 & 0.1715 & 0.1715 \\
\hline & & & & 1.5 & & & & & 0.5503 & 0.5503 & 0.2595 & 0.2595 \\
\hline & & & & & 1.2 & & & & 1.0690 & 1.0690 & 0.0861 & 0.0861 \\
\hline & & & & & 1.4 & & & & 1.1597 & 1.1597 & 0.0106 & 0.0106 \\
\hline & & & & & 1.6 & & & & 1.2430 & 1.2430 & -0.0598 & -0.0598 \\
\hline & & & & & & 0.5 & & & 0.8188 & 0.8188 & 0.2987 & 0.2987 \\
\hline & & & & & & 1 & & & 0.6332 & 0.6332 & 0.4600 & 0.4600 \\
\hline & & & & & & 1.5 & & & 0.4499 & 0.4499 & 0.6191 & 0.6191 \\
\hline & & & & & & & 0.5 & & 0.9131 & 0.9131 & 0.2136 & 0.2136 \\
\hline & & & & & & & 1 & & 0.8378 & 0.8378 & 0.2757 & 0.2757 \\
\hline & & & & & & & 1.5 & & 0.7589 & 0.7589 & 0.3411 & 0.3411 \\
\hline & & & & & & & & 0.5 & 0.8446 & 0.8446 & 0.2758 & 0.2758 \\
\hline & & & & & & & & 1 & 0.7281 & 0.7281 & 0.3731 & 0.3731 \\
\hline & & & & & & & & 1.5 & 0.6421 & 0.6421 & 0.4262 & 0.4262 \\
\hline
\end{tabular}

TABLE 3: Behavior of various flow rate on local Nusselt number and Sherwood number by fixing $\lambda_{M}=0, M=k=I=\lambda_{T}=E c=N t=N b$ $=0.1$, and $\operatorname{Pr}=1$.

\begin{tabular}{|c|c|c|c|c|c|c|c|c|}
\hline \multirow[b]{2}{*}{$Q$} & \multirow[b]{2}{*}{$L e$} & \multirow[b]{2}{*}{$\chi$} & \multirow[b]{2}{*}{$\beta$} & \multirow[b]{2}{*}{$V_{r}$} & \multicolumn{2}{|c|}{$N u_{x} R e_{x}^{-1 / 2}$} & \multicolumn{2}{|c|}{$S h_{x} R e_{x}^{-1 / 2}$} \\
\hline & & & & & shooting & bvp4c & shooting & bvp4c \\
\hline 0 & 0.5 & 0.1 & 1 & 1.5 & 0.9690 & 0.9690 & 0.1681 & 0.1681 \\
\hline 0.1 & & & & & 1.1504 & 1.1504 & 0.1184 & 0.1184 \\
\hline 0.15 & & & & & 1.3522 & 1.3522 & 0.1121 & 0.1121 \\
\hline 0.2 & & & & & 1.5539 & 1.5539 & -0.1084 & -0.1084 \\
\hline & 1 & & & & 0.9554 & 0.9554 & 0.6604 & 0.6604 \\
\hline & 1.5 & & & & 0.9475 & 0.9475 & 1.0198 & 1.0198 \\
\hline & 3 & & & & 0.9350 & 0.9350 & 1.7924 & 1.7924 \\
\hline & & 0.5 & & & 0.9588 & 0.9588 & 0.5295 & 0.5295 \\
\hline & & 2 & & & 0.9436 & 0.9436 & 1.2736 & 1.2736 \\
\hline & & 4 & & & 0.9350 & 0.9350 & 1.8908 & 1.8908 \\
\hline & & & 3 & & 0.9202 & 0.9202 & 0.1594 & 0.1594 \\
\hline & & & 5 & & 0.9061 & 0.9061 & 0.1581 & 0.1581 \\
\hline & & & 7 & & 0.8994 & 0.8994 & 0.1577 & 0.1577 \\
\hline & & & & 2 & 0.9987 & 0.9987 & 0.1765 & 0.1765 \\
\hline & & & & 2.5 & 1.0191 & 1.0191 & 0.1837 & 0.1837 \\
\hline & & & & 4 & 1.0551 & 1.0551 & 0.1989 & 0.1989 \\
\hline
\end{tabular}



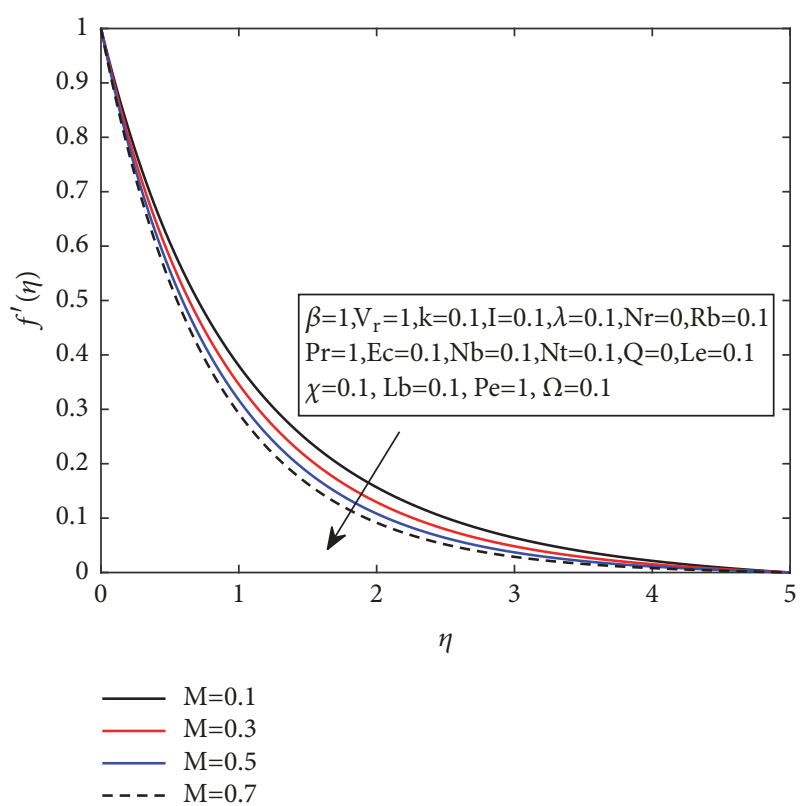

FIgURE 2: Variation of $M$ on $f^{\prime}$.

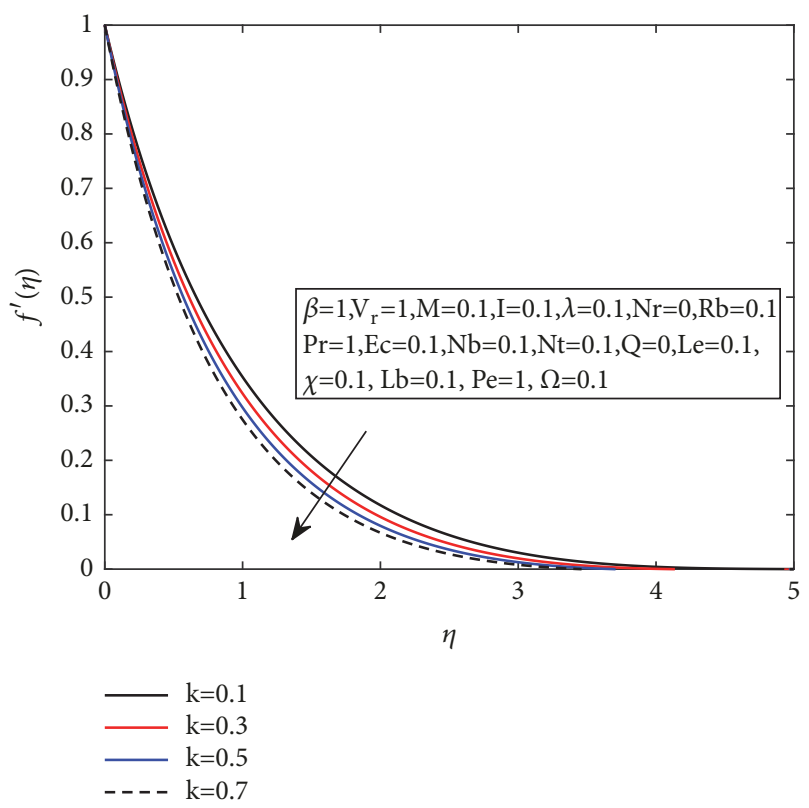

Figure 3: Behavior of $k$ on $f^{\prime}$.

Prandtl number exhibits the ratio of kinematic viscosity to thermal diffusivity [36-39], and the value of Prandtl number is considered about 0.7 for gasses while for water it is about 7. As temperature is increased, the value of Prandtl number declines; therefore we have considered the values of Prandtl number between 0.1 and 1.5. In heat transfer mechanism, the Prandtl number maintains the momentum and thermal boundary layers thickness. If we take the value of Pr small, it reflects that the heat diffusivity can be compared to the momentum of the flow in a short span of time. This indicates that, for the case of liquid metals, the thermal boundary layer thickness is much higher than to the boundary layer velocity.

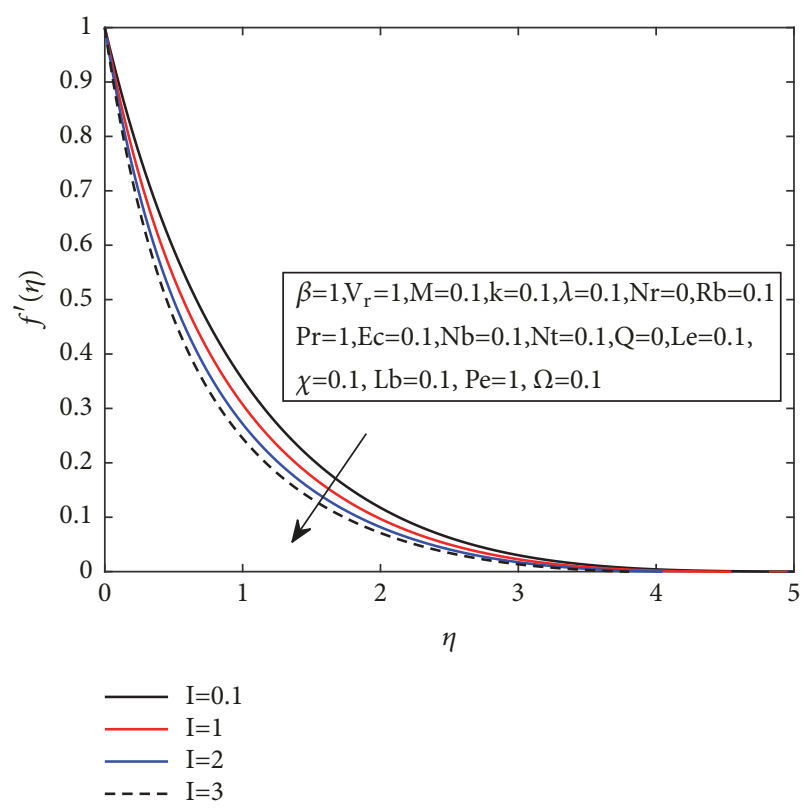

FIgURE 4: Illustrating the impact of $I$ on $f^{\prime}$.

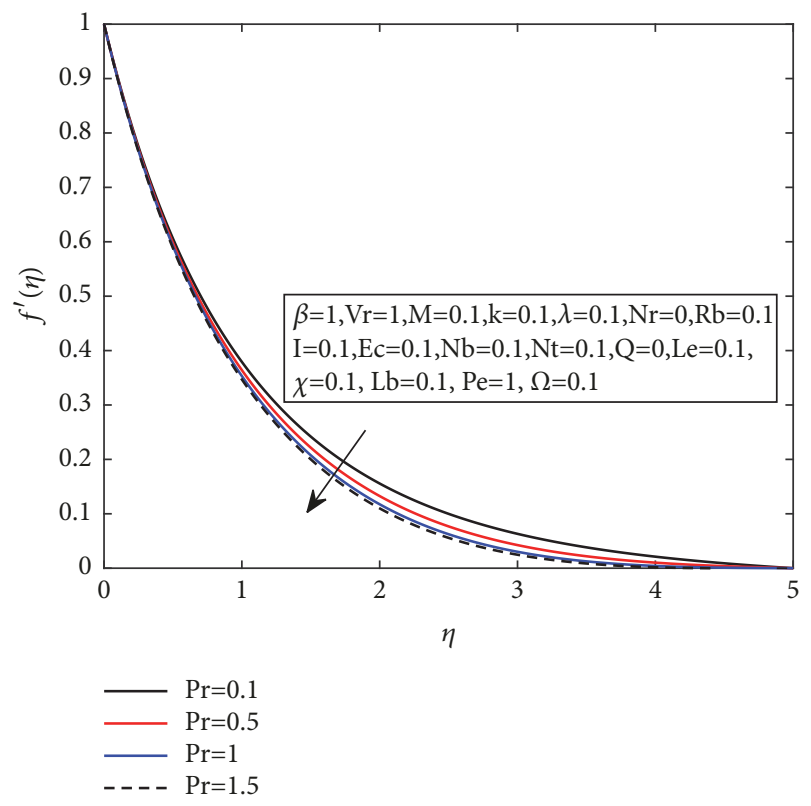

Figure 5: Demonstrating the effect of $\operatorname{Pr}$ on $f^{\prime}$.

So, by enhancing Prandtl number Pr temperature profile declines; on the other hand thermal boundary layer rises. By escalating the thermal diffusivity values, the boundary layer thickness declines because reduction is seen in the Prandtl number. Figure 6 indicates the variations of the thermal convective parameter $\lambda_{T}$ on the velocity. When increase in the thermal convective parameter values is made, a progressive increment in the flow velocity is seen. The impact of mass convective parameter $\lambda_{M}$ on the velocity profile is demonstrated in Figure 7. The principle factor is the Buoyancy forces that are responsible to reduce the velocity profile. By enhancing the value of $\lambda_{M}$, the velocity increases 

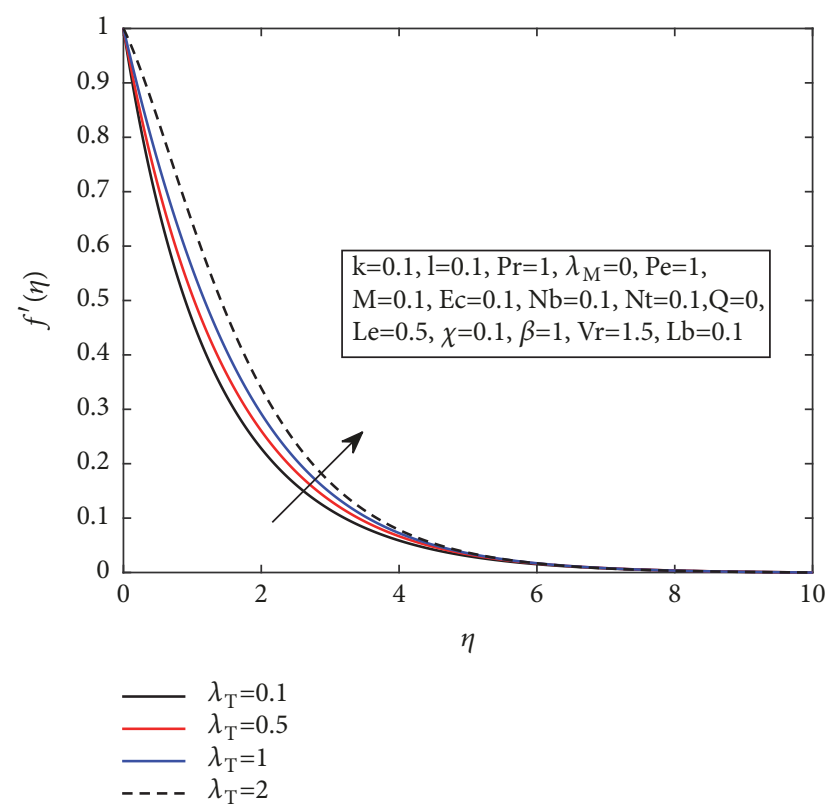

Figure 6: Demonstrating the effect of $\lambda_{T}$ on $f^{\prime}$.

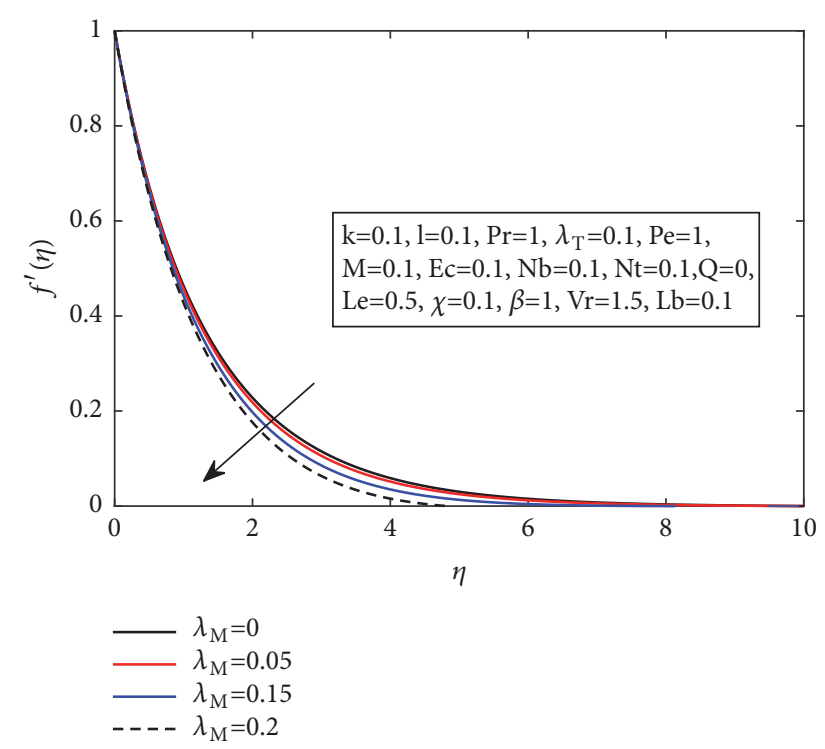

Figure 7: Variation of $\lambda_{M}$ on $f^{\prime}$ is exhibited.

within the boundary layer which brings a decrement in the velocity profile. Figure 8 reflects the impact of Brownian motion parameter $\mathrm{Nb}$ on the temperature profile. To enhance the $\mathrm{Nb}$ values, the intermolecular collision between the atoms is increased, which escalates the temperature of the fluid at the end. The variations of the thermophoresis parameter $\mathrm{Nt}$ on the temperature profile plots are highlighted in Figure 9. By escalating the thermophoresis parameter, the molecules move from hot surface to the cold one, which results in an enhancement in the thermal layer boundary, which decreases the temperature profile. The graphical effects of Lewis number $L e$ on the concentration profile are exhibited in Figure 10. Chen et al. [40] experimentally used the values of Lewis

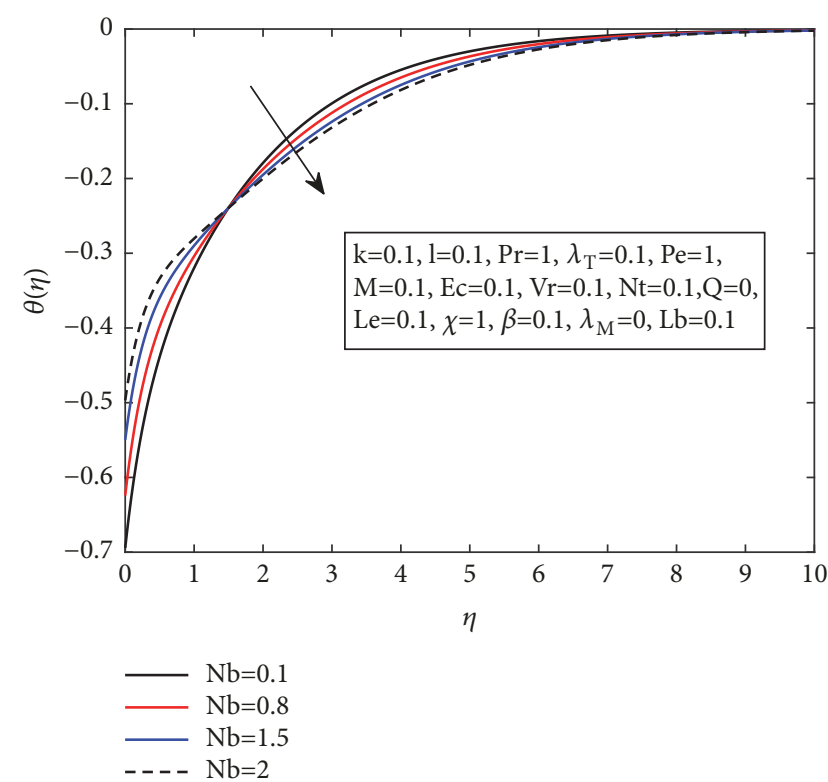

Figure 8: Variations of $\mathrm{Nb}$ on the temperature profile $\theta$.

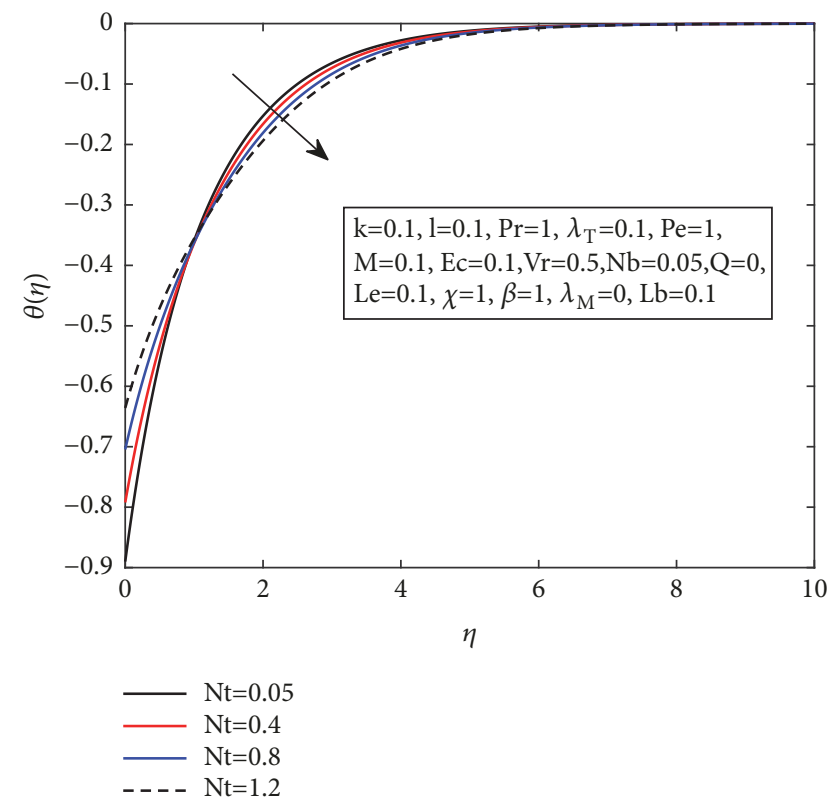

Figure 9: Demonstrating the effect of $N t$ on $\theta$.

number between 0 and 2. Various researchers [27, 40-42] have studied stretching sheet phenomena theoretically and experimentally and used similar ranges in their studies. Decrease in the velocity profile is recorded close to the boundary wall and an enhancement is seen by moving away from the boundary. The evidence behind this process is the occurrence of the boundary layer thickness that increases the nanoparticle volume fraction and its boundary layer. Figure 11 highlights the impacts of the chemical reaction parameter $\chi$ on the nanoparticle concentration profile. It depicts that the concentration profile decreases near the boundary wall and the concentration of the species escalates by moving away 

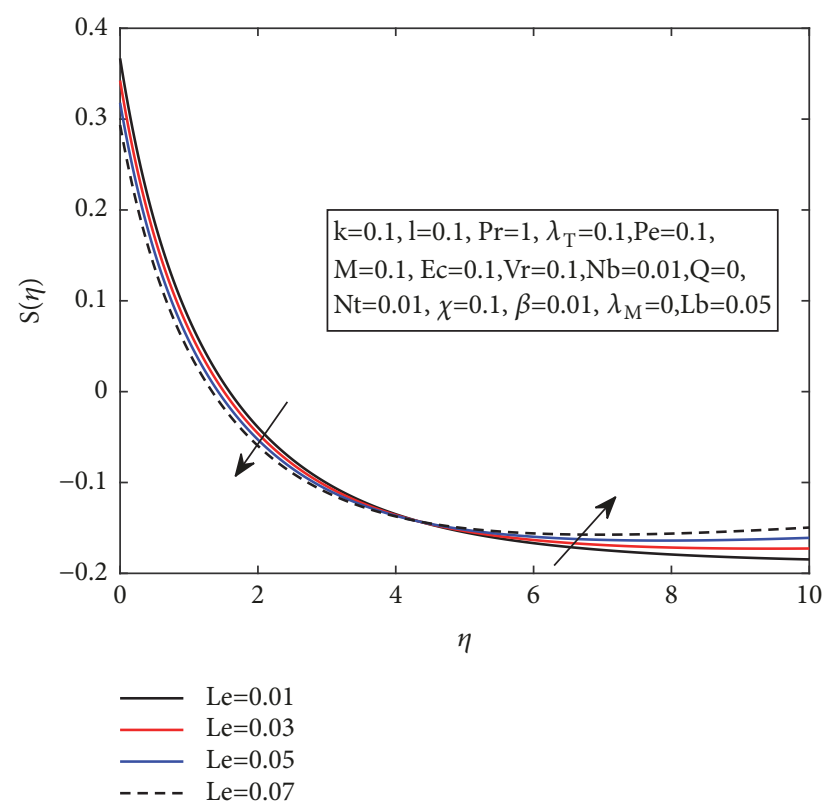

FIgURE 10: Illustrating the effect of $L e$ on S.

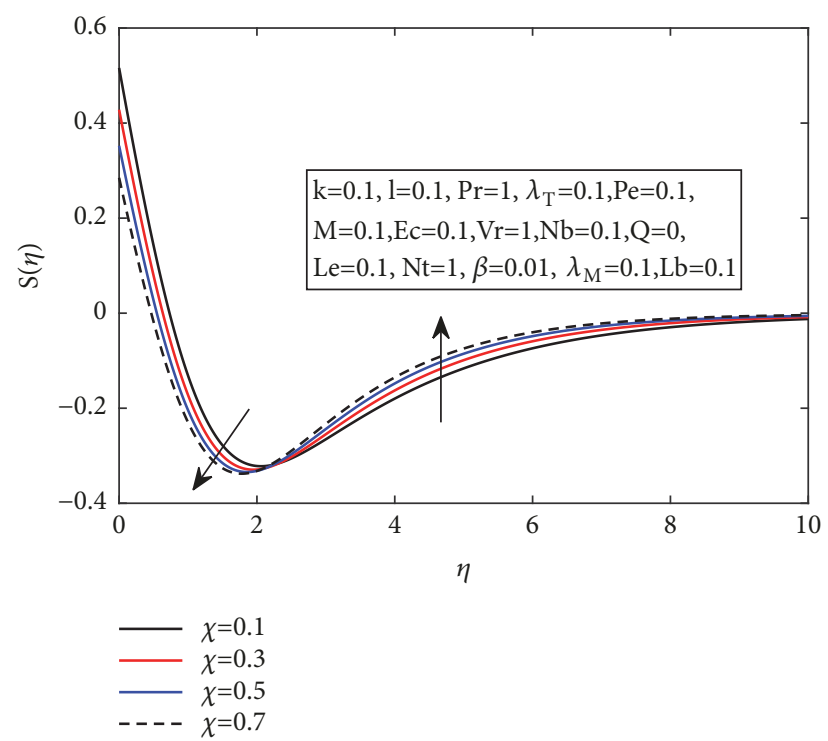

FIgURE 11: Demonstrating the effect of $\chi$ on $S$.

from the boundary wall. The effects of the thermophoresis parameter $N t$ on the nanoparticles concentration profile are depicted in Figure 12. It is concluded that huge gap in temperature drives the fluid more quickly from hot surface to the cold. And finally, the depth of the concentration boundary layer increases which ultimately augments the concentration profile. Figure 13 represents the behavior of the mass friction field $S$ versus the Brownian motion parameter $N b$. By enhancing the Brownian motion parameter, the thickness of thermal boundary layer increases due to this concentration profile. Figure 14 displays the behavior of the bioconvection Lewis number parameter $L b$ against the density of motile microorganisms profile. An increment in the value of $L b$ leads
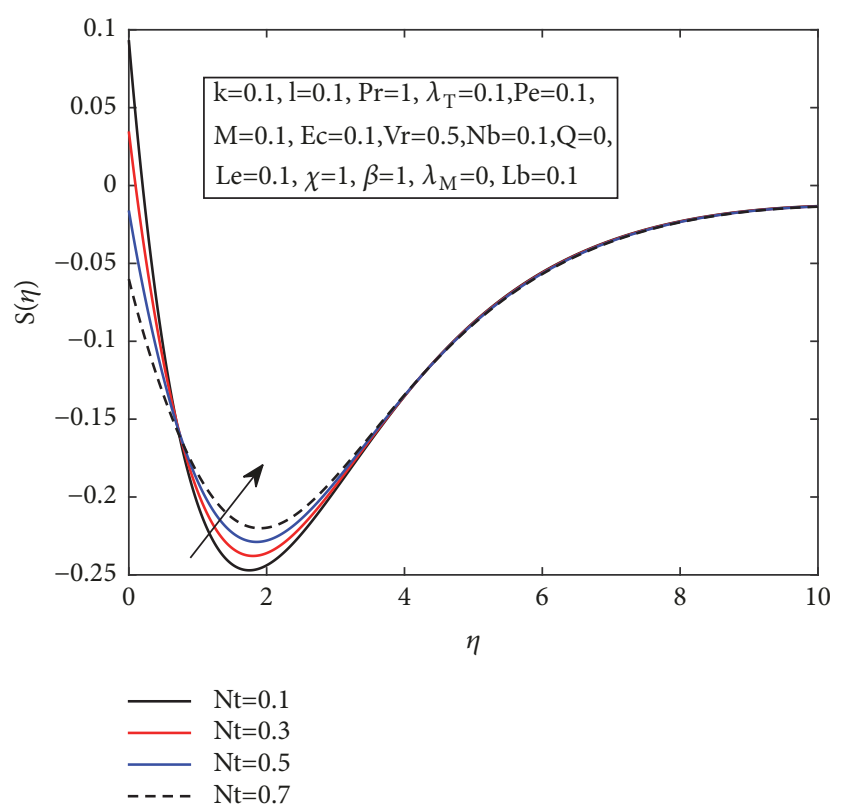

FIgURE 12: Illustrating the effect of $N t$ on $S$.

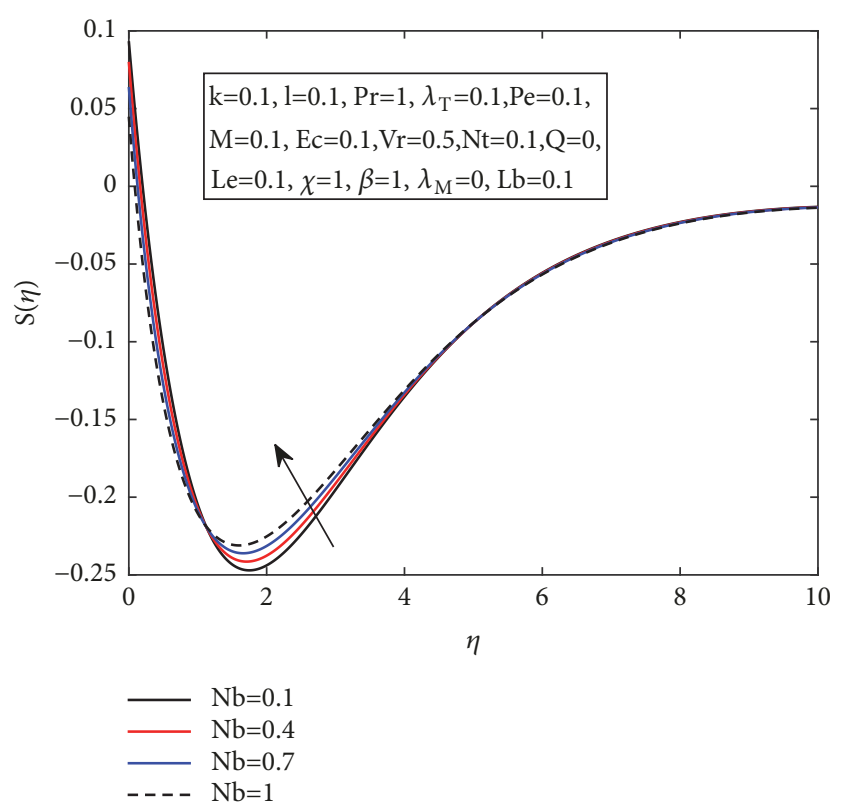

Figure 13: Demonstration of $\mathrm{Nb}$ on $\mathrm{S}$.

to a decrement of the microorganisms diffusion. Ultimately, thickness of the boundary layer along with density of the motile microorganisms declines.

\section{Conclusion}

The current investigation is designed to solve the two-phase Casson nanofluid passing over a stretching sheet using the permeable surface along with the effects of the chemical reactions and gyrotactic microorganisms. The systems of nonlinear PDEs are transformed to ODEs by employing the 


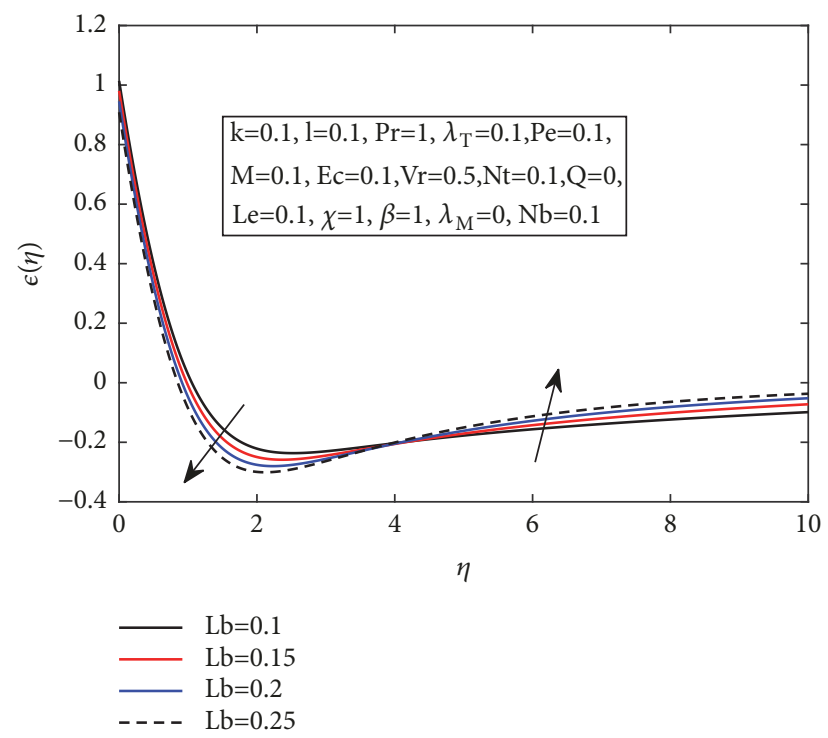

FIgURE 14: Behavior of $L b$ on $\epsilon$.

similarity transformations. The well known shooting numerical technique along with the fourth order Runge-Kutta numerical approach is deployed to solve these ODEs. The software used for solving this system of nonlinear equations is MATLAB R2016(a) package. Some novel findings of this investigation are presented as follows:

(i) It is evident from this study that Local Nusselt number rises with the enhancement in magnetic field parameter, the porous media parameter, and the chemical reactions.

(ii) The magnetic field parameter $M$ along with porous media parameter $k$ shows a decreasing effect on the velocity profile $f^{\prime}(\eta)$.

(iii) The inertia parameter $I$ and the Prandtl number $P r$ demonstrate a decreasing effect on the velocity profile $f^{\prime}(\eta)$.

\section{Nomenclature}

$B_{0}$ : Uniform magnetic field

$u, v$ : Velocity components

$\rho: \quad$ Density of the fluid

$v$ : Kinematic viscosity

$\widetilde{v}$ : Reference kinematic viscosity

$k$ : Uneven permeability

$C_{p}$ : Heat at fixed pressure

$T: \quad$ Temperature of the fluid

$C$ : Concentration of the fluid

$c^{*}:$ Inertia value

$D_{\beta}:$ Brownian coefficients

$D_{t}$ : Thermophoresis diffusion coefficient

$\sigma$ : Electrical conductivity

$u_{0}$ : Reference velocity

$T_{0}$ : Reference temperature

$C_{0}$ : Concentration

$l$ : Length $\epsilon: \quad$ Gyrotactic microorganisms

$Q_{0}$ : Heat generation coefficients

$K_{0}$ : Chemical reaction coefficient

$\beta$ : Casson fluid factor

$V_{r}$ : Viscosity ratio factor

$I: \quad$ Inertia factor

$\lambda_{T}$ : Thermal convective factor

$\lambda_{M}$ : Mass convective factor

$P e$ : Bioconvection Peclet number

$M$ : Magnetic field factor

Pr: Prandtl number

$N b$ : Brownian motion factor

$N t$ : Thermophoresis factor

Le: Lewis number

Q: Heat generation/absorption factor

$\chi$ : Chemical reaction factor

Ec: Eckert number

$L b$ : Bioconvection Lewis number

$\Omega:$ Microorganism concentration difference parameter.

\section{Data Availability}

All the data used in this research work is available within the manuscript.

\section{Conflicts of Interest}

The authors declare that they have no conflicts of interest.

\section{References}

[1] S. Mirmasoumi and A. Behzadmehr, "Numerical study of laminar mixed convection of a nanofluid in a horizontal tube using two-phase mixture model," Applied Thermal Engineering, vol. 28, no. 7, pp. 717-727, 2008.

[2] K. Das, "Slip flow and convective heat transfer of nanofluids over a permeable stretching surface," Computers \& Fluids, vol. 64, pp. 34-42, 2012.

[3] W. A. Khan and I. Pop, "Boundary-layer flow of a nanofluid past a stretching sheet," International Journal of Heat and Mass Transfer, vol. 53, no. 11-12, pp. 2477-2483, 2010.

[4] R. S. Gorla and A. Chamkha, "Natural convective boundary layer flow over a horizontal plate embedded in a porous medium saturated with a nanofluid," Journal of Modern Physics, vol. 02, no. 02, pp. 62-71, 2011.

[5] A. Noghrehabadi, R. Pourrajab, and M. Ghalambaz, "Effect of partial slip boundary condition on the flow and heat transfer of nanofluids past stretching sheet prescribed constant wall temperature," International Journal of Thermal Sciences, vol. 54, pp. 253-261, 2012.

[6] A. Noghrehabadi, R. Pourrajab, and M. Ghalambaz, "Flow and heat transfer of nanofluids over stretching sheet taking into account partial slip and thermal convective boundary conditions," Heat and Mass Transfer, vol. 49, no. 9, pp. 13571366, 2013.

[7] W. Ibrahim and B. Shankar, "MHD boundary layer flow and heat transfer of a nanofluid past a permeable stretching sheet with velocity, thermal and solutal slip boundary conditions," Computers \& Fluids, vol. 75, pp. 1-10, 2013. 
[8] M. Goyal and R. Bhargava, "Boundary layer flow and heat transfer of viscoelastic nanofluids past a stretching sheet with partial slip conditions," Applied Nanoscience, vol. 4, no. 6, pp. 761-767, 2014.

[9] M. R. Krishnamurthy, B. C. Prasannakumara, B. J. Gireesha, and R. S. R. Gorla, "Effect of chemical reaction on MHD boundary layer flow and melting heat transfer of Williamson nanofluid in porous medium," Engineering Science and Technology, an International Journal, vol. 19, no. 1, pp. 53-61, 2016.

[10] M. Sheikholeslami, T. Hayat, and A. Alsaedi, "MHD free convection of $\mathrm{Al} 2 \mathrm{O} 3$-water nanofluid considering thermal radiation: A numerical study," International Journal of Heat and Mass Transfer, vol. 96, pp. 513-524, 2016.

[11] F. Garoosi, G. Bagheri, and F. Talebi, "Numerical simulation of natural convection of nanofluids in a square cavity with several pairs of heaters and coolers (HACs) inside," International Journal of Heat and Mass Transfer, vol. 67, pp. 362-376, 2013.

[12] M. Turkyilmazoglu, "An analytical treatment for the exact solutions of MHD flow and heat over two-three dimensional deforming bodies," International Journal of Heat and Mass Transfer, vol. 90, pp. 781-789, 2015.

[13] J. Buongiorno, "Convective transport in nanofluids," Journal of Heat Transfer, vol. 128, no. 3, pp. 240-250, 2006.

[14] P. M. Congedo, S. Collura, and P. M. Congedo, "Modeling and analysis of natural convection heat transfer in nanofluids," in Proceedings of the ASME Summer Heat Transfer Conference, vol. 3, pp. 569-579, August 2008.

[15] M. M. Rashidi, S. Abelman, and N. Freidoonimehr, "Entropy generation in steady MHD flow due to a rotating porous disk in a nanofluid," International Journal of Heat and Mass Transfer, vol. 62, no. 1, pp. 515-525, 2013.

[16] K. Vafai, Handbook of Porous Media, CRC Press, 2015.

[17] A. Nakayama and A. V. Shenoy, "Non-darcy forced convective heat transfer in a channel embedded in a non-newtonian inelastic fluid-saturated porous medium," The Canadian Journal of Chemical Engineering, vol. 71, no. 1, pp. 168-173, 1993.

[18] D. A. Nield and A. Bejan, Convection in Porous Media, Springer, New York, NY, USA, 2006.

[19] A. Y. Bakier, "Thermal radiation effect on mixed convection from vertical surfaces in saturated porous media," International Communications in Heat and Mass Transfer, vol. 28, no. 1, pp. 119-126, 2001.

[20] W. A. Khan, O. D. Makinde, and Z. H. Khan, "MHD boundary layer flow of a nanofluid containing gyrotactic microorganisms past a vertical plate with Navier slip," International Journal of Heat and Mass Transfer, vol. 74, pp. 285-291, 2014.

[21] H. Xu and I. Pop, "Mixed convection flow of a nanofluid over a stretching surface with uniform free stream in the presence of both nanoparticles and gyrotactic microorganisms," International Journal of Heat and Mass Transfer, vol. 75, pp. 610623, 2014.

[22] A. V. Kuznetsov, "The onset of nanofluid bioconvection in a suspension containing both nanoparticles and gyrotactic microorganisms," International Communications in Heat and Mass Transfer, vol. 37, no. 10, pp. 1421-1425, 2010.

[23] A. V. Kuznetsov and D. A. Nield, "The Cheng-Minkowycz problem for natural convective boundary layer flow in a porous medium saturated by a nanofluid: a revised model," International Journal of Heat and Mass Transfer, vol. 65, pp. 682685, 2013.
[24] H. Xu and I. Pop, "Fully developed mixed convection flow in a horizontal channel filled by a nanofluid containing both nanoparticles and gyrotactic microorganisms," European Journal of Mechanics - B/Fluids, vol. 46, pp. 37-45, 2014.

[25] A. Mahdy, "Natural convection boundary layer flow due to gyrotactic microorganisms about a vertical cone in porous media saturated by a nanofluid," Journal of the Brazilian Society of Mechanical Sciences and Engineering, vol. 38, no. 1, pp. 67-76, 2016.

[26] N. S. Akbar, "Bioconvection peristaltic flow in an asymmetric channel filled by nanofluid containing gyrotactic microorganism: bio nano engineering model," International Journal of Numerical Methods for Heat \& Fluid Flow, vol. 25, no. 2, pp. 214224, 2015.

[27] T. Hayat, S. A. Shehzad, and A. Alsaedi, "Soret and Dufour effects on magnetohydrodynamic (MHD) flow of Casson fluid," Applied Mathematics and Mechanics, vol. 33, no. 10, pp. 13011312, 2012.

[28] P. K. Kameswaran, M. Narayana, P. Sibanda, and P. V. S. N. Murthy, "Hydromagnetic nanofluid flow due to a stretching or shrinking sheet with viscous dissipation and chemical reaction effects," International Journal of Heat and Mass Transfer, vol. 55, no. 25-26, pp. 7587-7595, 2012.

[29] J. Alinejad and S. Samarbakhsh, "Viscous flow over nonlinearly stretching sheet with effects of viscous dissipation," Journal of Applied Mathematics, vol. 2012, Article ID 587834, 10 pages, 2012.

[30] S. U. S. Choi, "Enhancing thermal conductivity of fluids with nanoparticles," in Developments and Applications of NonNewtonian Ows, D. A. Siginer and H. P. Wang, Eds., vol. 66, pp. 99-105, ASME, New York, NY, USA, 1995.

[31] W. Yu, H. Xie, L. Chen, and Y. Li, "Investigation on the thermal transport properties of ethylene glycol-based nanofluids containing copper nanoparticles," Powder Technology, vol. 197, no. 3, pp. 218-221, 2010.

[32] M. R. Eid, "Chemical reaction effect on MHD boundarylayer flow of two-phase nanofluid model over an exponentially stretching sheet with a heat generation," Journal of Molecular Liquids, vol. 220, pp. 718-725, 2016.

[33] M. Waqas, T. Hayat, S. A. Shehzad, and A. Alsaedi, “Transport of magnetohydrodynamic nanomaterial in a stratified medium considering gyrotactic microorganisms," Physica B: Condensed Matter, vol. 529, pp. 33-40, 2018.

[34] M. Ferdows, M. Shakhaoath Khan, M. M. Alam, and S. Sun, "MHD mixed convective boundary layer flow of a nanofluid through a porous medium due to an exponentially stretching sheet," Mathematical Problems in Engineering, vol. 2012, Article ID 408528, 21 pages, 2012.

[35] B. Bidin and R. Nazar, "Numerical solution of the boundary layer flow over an exponentially stretching sheet with thermal radiation," European Journal of Scientific Research, vol. 33, no. 4, pp. 710-717, 2009.

[36] T. L. Bergman, A. S. Lavine, and F. P. Incropera, Fundamentals of Heat and Mass Transfer, John Wiley \& Sons, Incorporated, 7th edition, 2011.

[37] A. Yunus, Cengel, Heat and Mass Transfer, McGraw-Hill Education, 2011.

[38] C. P. Kothandaraman, Fundamentals of Heat and Mass Transfer, New Age International, 2006.

[39] U.S. Department of Energy, Thermodynamics, Heat Transfer and Fluid Flow, vol. 2 of 3, DOE Fundamentals Handbook, 2016. 
[40] R.-H. Chen, M. Chaos, and A. Kothawala, "Lewis number effects in laminar diffusion flames near and away from extinction," Proceedings of the Combustion Institute, vol. 31, no. 1, pp. 1231-1237, 2007.

[41] N. S. Akbar and Z. H. Khan, "Magnetic field analysis in a suspension of gyrotactic microorganisms and nanoparticles over a stretching surface," Journal of Magnetism and Magnetic Materials, vol. 410, pp. 72-80, 2016.

[42] A. Zaib, K. Bhattacharyya, M. S. Uddin, and S. Shafie, "Dual Solutions of non-newtonian casson fluid flow and heat transfer over an exponentially permeable shrinking sheet with viscous dissipation," Modelling and Simulation in Engineering, vol. 2016, Article ID 6968371, 8 pages, 2016. 


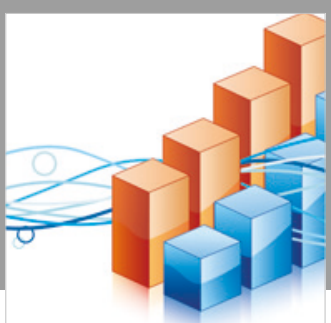

Advances in

Operations Research

\section{-n-m}
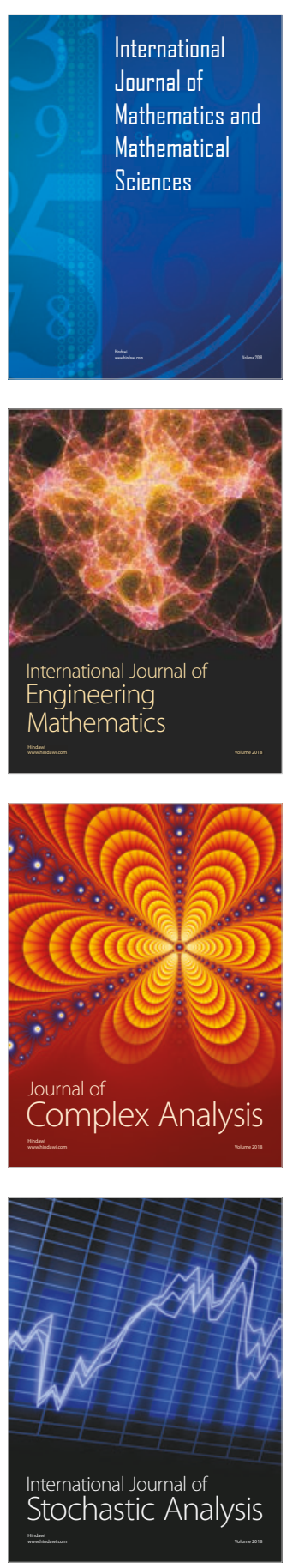
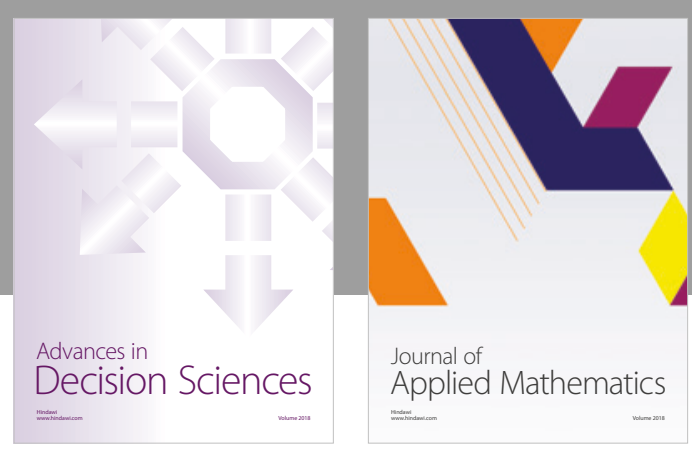

Journal of

Applied Mathematics
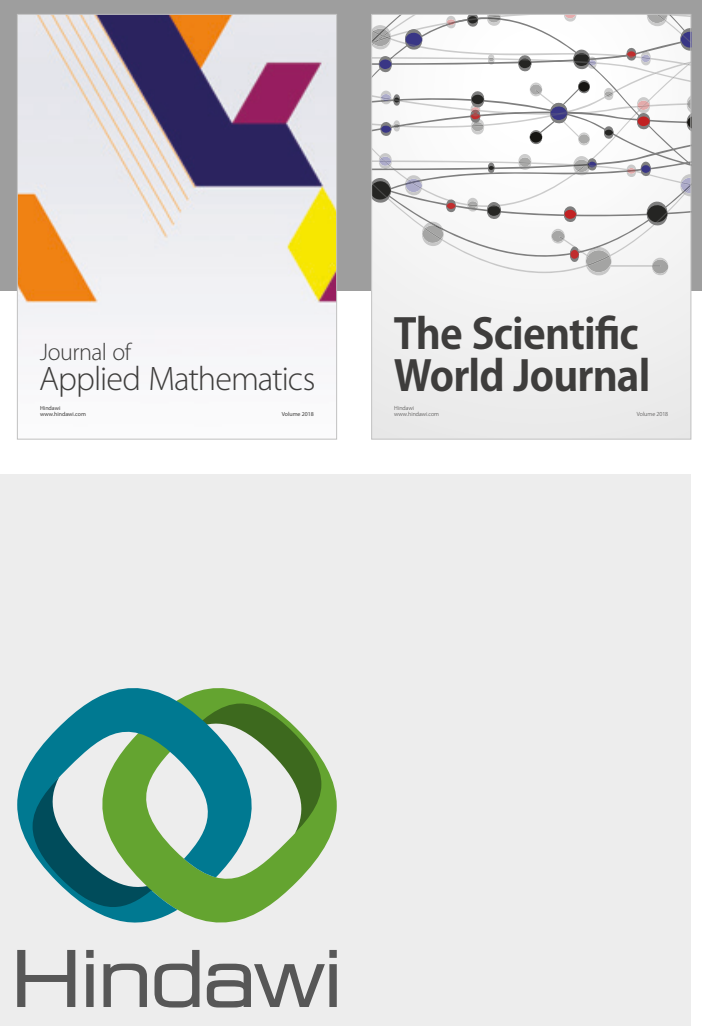

Submit your manuscripts at

www.hindawi.com

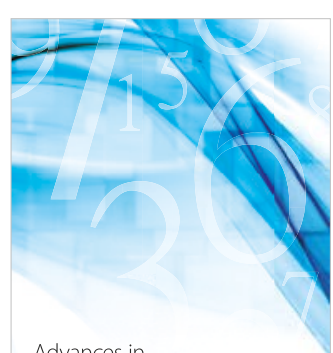

Advances in
Numerical Analysis
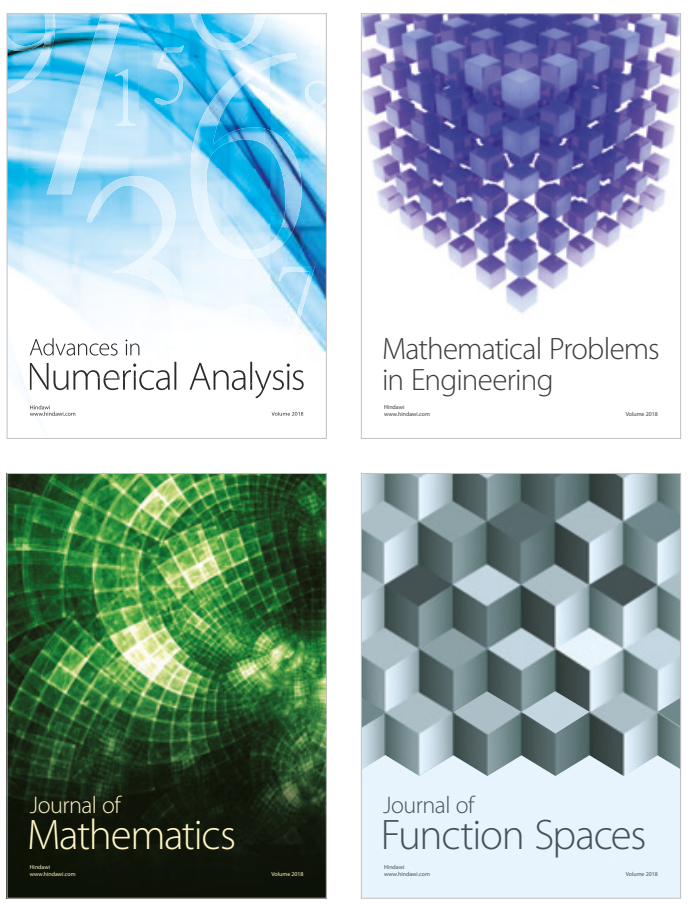

Mathematical Problems in Engineering

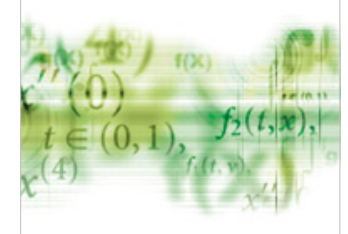

International Journal of

Differential Equations

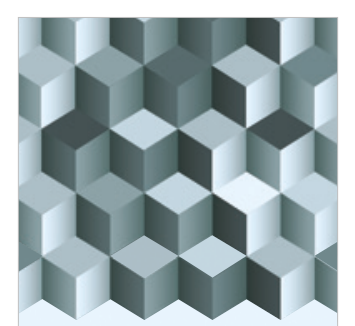

Journal of

Function Spaces

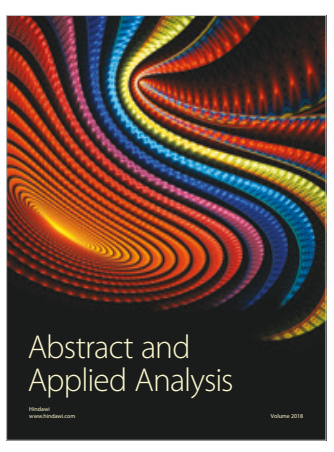

The Scientific

World Journal

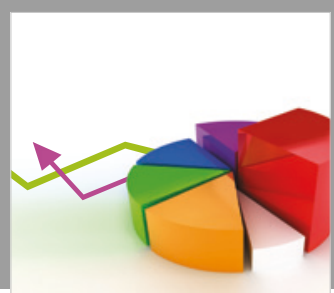

Journal of

Probability and Statistics
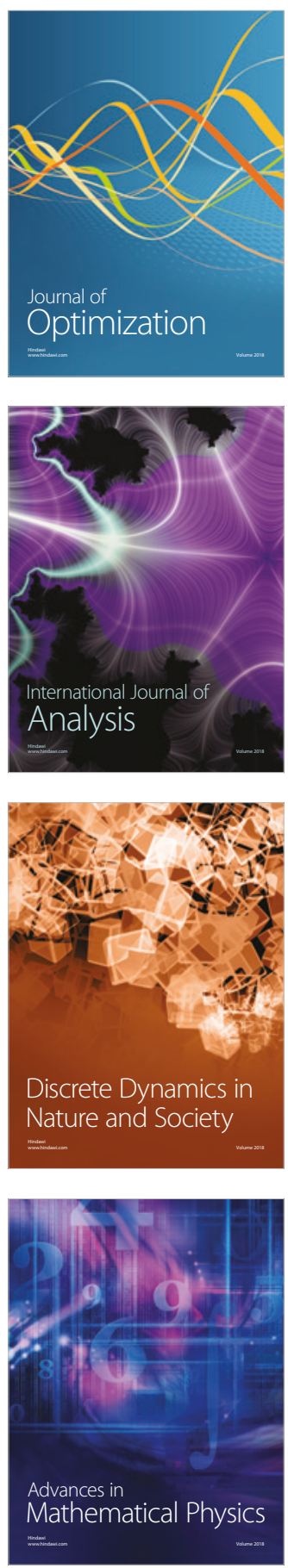